\title{
De la atención de un brote por un patógeno desconocido en Wuhan hasta la preparación y respuesta ante la emergencia de Covid-19 en México
}

Eduardo López-Ortiz, ${ }^{1}$ Geovani López-Ortiz, ${ }^{1}$ Indira R. Mendiola-Pastrana, ${ }^{1}$ Juan J. Mazón-Ramírez ${ }^{1}$ y José A. Díaz-Quiñonez*

${ }^{1}$ Subdivisión de Medicina Familiar; ${ }^{2}$ División de Estudios de Posgrado. Universidad Nacional Autónoma de México, Facultad de Medicina, Ciudad de México, México

\section{Resumen}

El 31 de diciembre de 2019, las autoridades chinas de salud informaron a la comunidad internacional, a través de los mecanismos establecidos por la Organización Mundial de la Salud (OMS), de una epidemia de neumonía con etiología desconocida en Wuhan, provincia de Hubei. Los primeros casos se notificaron a inicios de ese mes y se vincularon al antecedente de visitar un mercado de comida y animales vivos. El 7 de enero de 2020 se logró el aislamiento y reconocimiento del patógeno responsable mediante secuenciación de siguiente generación, mientras el número de afectados continuaba en ascenso. La publicación de genomas completos del nuevo coronavirus identificado (inicialmente denominado 2019-nCoV, ahora designado SARS-CoV2) en bases de datos públicas y privadas, de protocolos diagnósticos estandarizados y de la información clínica epidemiológica generada permitirá atender la Emergencia de Salud Pública de Importancia Internacional (ESPII) declarada el 30 de enero por la OMS. Con este documento pretendemos aportar a la caracterización de la epidemia de neumonía, ahora llamada enfermedad por coronavirus (Covid-19), revisar las fortalezas que tiene México en el concierto de la salud global e invitar a los profesionales de la salud a incorporarse a las actividades de preparación y respuesta ante esta emergencia.

PALABRAS CLAVE: Nuevo coronavirus. SARS-CoV2. Covid-19. México.

\section{From the handling of an outbreak by an unknown pathogen in Wuhan to the preparedness and response in the face of the emergence of Covid-19 in Mexico}

\begin{abstract}
On December 31, 2019, the Chinese health authorities informed the international community, through the mechanisms established by the World Health Organization (WHO), of a pneumonia epidemic of unknown etiology in Wuhan, Hubei Province. The first cases were reported early in that month and were linked to a history of having visited a market where food and live animals are sold. On January 7, 2020, isolation and identification of the culprit pathogen was achieved using next-generation sequencing, while the number of affected subjects continued to rise. The publication of full-genomes of the newly identified coronavirus (initially called 2019-nCoV, now designated SARS-CoV2) in public and private databases, of standardized diagnostic protocols and of the clinical-epidemiological information generated will allow addressing the Public Health Emergency of International Concern (PHEIC), declared on January 30 by the WHO. With this document, we intend to contribute to the characterization of the pneumonia epidemic, now called Corona virus disease (Covid-19) review the strengths Mexico has in the global health concert and invite health professionals to join the preparedness and response activities in the face of this emergency.
\end{abstract}

KEY WORDS: New coronavirus. SARS-CoV2. Covid-19. Mexico.

Correspondencia:

*José A. Díaz-Quiñonez

E-mail: adiazq@unam.mx

DOI: 10.24875/GMM.20000046 CC BY-NC-ND (http://creativecommons.org/licenses/by-nc-nd/4.0/).
Fecha de recepción: 05-02-2020

Fecha de aceptación: 05-02-2020

www.gacetamedicademexico.com
Gac Med Mex. 2020;156:133-138

Disponible en PubMed

atículo open access bajo la licencia 


\section{Caracterización de la epidemia de neumonía de etiología desconocida}

Los coronavirus pertenecen a la familia Coronaviridae y se dividen en cuatro géneros: alfa, beta, gama y delta. Los dos primeros tienen la capacidad de infectar mamíferos, mientras que los gama y delta infectan principalmente aves, con cierto potencial de infectar mamíferos. ${ }^{1}$

Los alfa y beta coronavirus suelen desencadenar sintomatología respiratoria en humanos y gastrointestinal en otros mamíferos; casi todos los coronavirus que han afectado a humanos parecen provenir de los murciélagos. ${ }^{1,2}$

Las partículas de los coronavirus miden alrededor de $160 \mathrm{~nm}$ y su material genético está contenido en una hebra de ácido ribonucleico de 27 a $32 \mathrm{~kb}$. El extremo $5^{\prime}$ terminal codifica para 16 proteínas no estructurales relacionadas con los procesos de transcripción y replicación, mientras que el extremo $3^{\prime}$ terminal codifica para proteínas estructurales de membrana, spike, nucleocápside y envoltura. ${ }^{1}$

La patogenicidad de los virus depende, en gran medida, del tipo de receptor y de los órganos en los que se expresa. Respecto al coronavirus responsable del síndrome respiratorio agudo severo y el SARSCoV2, el receptor es la misma enzima convertidora de angiotensina 2 y otras proteasas con amplia distribución en tejidos pulmonares. ${ }^{3-5}$

El reporte de los primeros casos de esta epidemia se puede rastrear al 8 de diciembre de 2019. Del análisis clínico molecular de muestras obtenidas de pulmón, raspado nasofaríngeo y sangre de 42 pacientes que presentaron sintomatología respiratoria con antecedente de visitar un mercado de animales, se obtuvieron 15 resultados positivos en la identificación de un coronavirus que no había sido observado, denominado hasta ese momento como 2019-nCov. ${ }^{2,6}$

Haber reconocido de forma temprana al agente patógeno como un coronavirus, con similitudes con el SARS-CoV (características microscópicas, antecedente de contacto con animales, sintomatología respiratoria), causante de un síndrome respiratorio agudo severo (SARS), facilitó la definición del sistema de vigilancia (el mismo que se usó durante el brote de 2003, responsable de más de 8000 casos en 26 países). ${ }^{6,7}$

La definición operacional que se empleó al inicio del brote en Wuhan se basó en las propuestas para la vigilancia y el reporte de los brotes de 2003 y 2012 e incluyó la presentación de cuatro criterios: fiebre (cuantificada o no), evidencia radiológica de afectación pulmonar, bajo conteo de leucocitos o linfocitos y ausencia de mejoría después de tres días de tratamiento antibiótico, o bien, la presentación de los tres primeros y el antecedente de contacto en el mercado de Wuhan. ${ }^{8-10}$

El 31 de diciembre, las autoridades de salud en China informaron sobre el brote a la comunidad internacional a través de la OMS, haciendo pública la información clínica epidemiológica preliminar. ${ }^{8,11,12}$

El 1 de enero de 2020, las autoridades sanitarias chinas cerraron el mercado vinculado con el brote para estudiar e identificar la fuente de contagio que continuaba siendo desconocida. ${ }^{6}$

El 7 de enero, mientras el número de afectados seguía en ascenso, las autoridades de salud en China lograron identificar al agente patógeno, información que se divulgó días después de la primera defunción, notificada el 9 de enero de 2020. El 12 de enero se compartió la secuencia del genoma del nuevo patógeno, seguido de otros depositados en las plataformas Genbank y en GISAID (Global Initiative on Sharing All Influenza Data). Fue el inicio del uso de protocolos estandarizados de detección del virus y con ello, la respuesta desde distintos sectores. ${ }^{13-16}$

El 13 y 15 de enero se reportaron los primeros casos importados en Tailandia y Japón, respectivamente; gradualmente otros territorios se fueron sumando al brote. ${ }^{17}$ Las autoridades en China decidieron colocar termómetros infrarrojos en aeropuertos y estaciones de camión y trenes.

Desde el 21 de enero, la OMS comenzó a publicar reportes de la situación en los países afectados. Entre las acciones de respuesta de las autoridades sanitarias de los países afectados, la OMS solicitó la búsqueda activa de casos y el seguimiento estrecho de contactos..$^{18}$ En esa misma fecha se publicó un análisis de riesgo de dispersión del virus y se alertó sobre los lugares a los que con mayor probabilidad podría dispersarse el virus, cálculos replicados más adelante con diferentes metodologías. ${ }^{19-22}$

El 23 de enero se reportó que, con los análisis filodinámicos a partir de 23 secuencias, no había evidencia de un reservorio intermedio entre la fuente de infección y los humanos. Estimaciones matemáticas calcularon el tamaño del brote en aproximadamente 4000 casos, cifra que hacía pensar que las cadenas de transmisión se mantenían por casos con sintomatología no tan severa que no buscaban atención médica. ${ }^{23,24}$

El 26 de enero, la evidencia de la transmisión entre humanos se fortaleció con uno de los casos en Vietnam.

El 30 de enero, la OMS anunció la situación como una nueva Emergencia de Salud Pública de 
Importancia Internacional (ESPII). En el reporte, se hizo especial énfasis en fortalecer las capacidades técnicas y de insumos en los laboratorios e infraestructura de salud. ${ }^{13,18,25}$ Una ESPII es un evento que representa riesgo de dispersión internacional de una enfermedad, la cual requiere una respuesta coordinada; la definición asume que la situación es grave, súbita, inusual e inesperada. ${ }^{26,27}$

Como ha sucedido con otras ESPII, la definición operacional ha sido dinámica. El 18 de enero se incluyó el antecedente de visita a Wuhan (independientemente de la visita al mercado cerrado el 1 de enero) o contacto con pacientes de Wuhan que habían tenido síntomas respiratorios en los últimos 14 días. Para la confirmación de los casos es necesario utilizar alguna técnica molecular: ${ }^{8,14}$

- Reacción en cadena de la polimerasa dirigida a identificar el marco de lectura 1a o 1b que codifica la proteína de nucleocápside del virus.

- Aislamiento de los virus.

- Hallazgo de información genética viral que relacione el caso con otras secuencias.

En el reporte del 31 de enero, el número de casos confirmados había superado al señalado para el brote de SARS en 2003 (Figura 1). Se publicó que de los 106 casos confirmados fuera de China, siete se identificaron asintomáticos. Para el 1 de febrero de 2020, la definición operacional para el brote define como sospechoso un paciente con infección respiratoria aguda severa con antecedente de fiebre y tos que requiera atención intrahospitalaria y cuya etiología no pueda ser explicada, además de lo siguiente: ${ }^{28}$

- Haber viajado o vivido en Wuhan en los 14 días anteriores a la presentación de síntomas.

- Ser un trabajador de la salud cuyas actividades se desarrollan en centros donde se atiende a personas con enfermedades respiratorias, independientemente de sus antecedentes de viaje o residencia.

Ese día se notificó que en Francia se había diagnosticado al primer trabajador de la salud fuera de China, además de compilarse información de Alemania y Japón que sustentó la existencia de transmisión entre humanos. Para el 5 de febrero, Bélgica se sumó a la lista de países que tienen casos confirmados. La OMS designó a la enfermedad como Covid-19 (acrónimo de Corona virus disease-2019) y lanzó un plan de preparación y respuesta de 675 millones de dólares como mecanismo para preparar a los países sin la infraestructura necesaria para enfrentar la emergencia. ${ }^{29}$

El 6 de febrero se reportó que 72 estados miembro de la OMS implementaron restricciones de viaje.

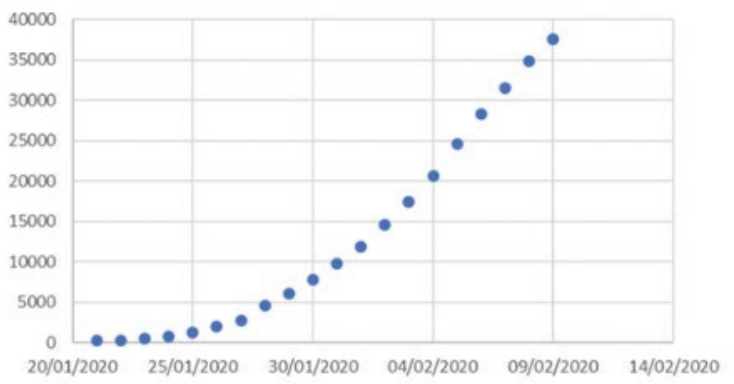

Figura 1. Casos acumulados de Covid-19 desde el inicio de los reportes diarios de la Organización Mundial de la Salud.

Notablemente, solo $32 \%$ de ellos notificó de forma oficial a la OMS. ${ }^{30}$

Para el 8 de febrero, con 24 países afectados, la respuesta de la OMS incluía la colaboración con diversos actores de la sociedad, desde sectores específicos como el financiero, agrícola, turístico, académico hasta público en general, tarea en la que el papel de las redes sociales ha sido central. ${ }^{31,32}$

El 9 de febrero, en su reporte diario, la OMS anunció que se están desarrollando proyectos dirigidos a la actualización sobre las medidas de protección del personal de salud, una herramienta de recolección de datos en campo y la traducción de materiales disponibles sobre el brote a varios idiomas. ${ }^{33}$

Los estimadores epidemiológicos para Covid-19 han variado conforme la información ha estado disponible, sin embargo, los cálculos del índice básico de reproducción continúan positivos, con periodos de incubación entre los dos y 14 días. Los hombres se mantienen con mayor proporción entre los diagnosticados (71\%), las personas de edad avanzada con comorbilidades representan el grupo poblacional más susceptible para morir por la infección y la mediana de edad al diagnóstico está alrededor de los 45 años. , $34-37^{2}$

\section{Preparación y respuesta en México}

La Secretaría de Salud de México ha puesto a disposición del público material de difusión con información general sobre la situación del brote de Covid-19 desde su inicio. El día 30 de enero emitió un aviso preventivo de viaje hacia la provincia de Hubei, China, y diariamente publica un reporte con datos verificados a partir de fuentes oficiales. ${ }^{38}$

Además, el Sistema Nacional de Vigilancia Epidemiológico elaboró el Lineamiento estandarizado para la vigilancia epidemiológica y por laboratorio de enfermedad por 2019-nCoV, documento que establece las definiciones operacionales, manejo de muestras, medidas de prevención y control de 
riesgos, además del algoritmo diagnóstico ante el riesgo de introducción del patógeno emergente. ${ }^{39}$ Este documento considera distintas esferas de la vigilancia epidemiológica ante una emergencia internacional y como anexo presenta un formato especial para el estudio de casos, seguimiento de contactos, recomendaciones en los puntos de entrada internacionales y una línea de acción para los trabajadores de la salud que atiendan un caso sospechoso.

La importancia de continuar con las actualizaciones de este lineamiento, la primera fue el 7 de febrero de 2020, es asegurar la rápida detección de los casos importados y de esta manera evitar la generación de cadenas de transmisión secundarias. ${ }^{39}$

En México se considera caso sospechoso de Covid-19 a una persona de cualquier edad que presente enfermedad respiratoria aguda y que cuente con el antecedente de viaje o estancia a la provincia de Hubei, China, o haber estado en contacto con un caso confirmado o bajo investigación hasta 14 días antes del inicio de síntomas. La confirmación se establece mediante métodos estandarizados por el Instituto de Diagnóstico y Referencia Epidemiológicos "Dr. Manuel Martínez Báez" (InDRE).

La investigación del caso sospechoso de Covid-19 se debe realizar con apego a las medidas de protección estándar y basadas en la transmisión (por gota y contacto):

- Precauciones estándar: lavado de manos (agua y jabón) o higiene de manos (alcohol en gel en concentraciones mayores de $70 \%$ en los cinco momentos definidos por la OMS), uso de guantes, uso de mascarilla con protección facial y de bata impermeable si existe riesgo de salpicaduras en ojos y cara, transporte de ropa contaminada en bolsa roja, uso de contenedores rígidos para el material punzocortante y nunca reencapuchar las agujas una vez utilizadas.

- Precaución de transmisión por gotas: mantener una distancia de un metro con el paciente, mantener puerta cerrada durante la atención, monitoreo de los visitantes, empleo personalizado de equipo médico como estetoscopio, termómetros o manguitos para la toma de presión arterial, si esto no es posible limpiar y desinfectar entre cada paciente con alcohol etílico a 70 \%; asignación de una habitación individual para el caso sospechoso 0 aislamiento de la cohorte, traslados del paciente solo por situaciones especiales y notificación al equipo de atención sobre estas medidas.

- Precaución de transmisión por aerosoles: uso de mascarilla N95 en procedimientos que generen aerosoles como aspiraciones, intubaciones, broncoscopias y reanimación cardiopulmonar.

Se han establecido líneas de trabajo para el personal de salud en contacto con los casos sospechosos, con diferencias para los niveles de atención existentes en México.

Ante la identificación de un caso que cumpla con el antecedente de viaje o contacto de riesgo, la primera medida del médico de atención primaria será proporcionar una mascarilla quirúrgica al paciente. A partir de ese momento, el médico valorará al paciente en un espacio aislado con medidas de protección como bata desechable, gafas y guantes y decidirá si cumple con la definición operacional; en caso positivo, empleará la mascarilla N95 como protección y realizará la toma de muestras y notificación a la instancia epidemiológica correspondiente. Si fuera necesaria la hospitalización, el paciente deberá ser enviado al nivel que amerite, monitoreado por el área de epidemiología.

En el segundo y tercer nivel de atención se asume que los pacientes llegarán con datos de dificultad respiratoria, ante lo cual el flujo de trabajo sería similar al del primer nivel. En estos niveles serán necesarias camas aisladas para los pacientes y el uso de equipo de protección especializado para el personal de salud, como mascarilla, bata desechable y gafas de protección, estas últimas debido a que hay evidencia de transmisión potencial por esa vía. ${ }^{40}$ El equipo de epidemiología figura como enlace para aportar información sobre el seguimiento de cada caso.

Las muestras para el diagnóstico de Covid-19 son exudados nasofaríngeo y faríngeo tomados con hisopos de dacrón o rayón, transportando los tubos juntos (en medio viral a $4{ }^{\circ} \mathrm{C}$ ) para incrementar la carga viral. Si el paciente se encuentra intubado, se deberá gestionar lavado bronquioalveolar (se requieren al menos $1 \mathrm{~mL}$ de aspirado y $1 \mathrm{~mL}$ de medio de transporte) y, en caso de defunción, biopsia pulmonar $\left(2 \mathrm{~cm}^{3}\right)$. Las muestras deben ser obtenidas durante los primeros cinco días de instauración del cuadro clínico y ser transportadas en sistema de triple embalaje, de acuerdo con los lineamientos establecidos..$^{39} \mathrm{Si}$ el caso se confirma, se recomienda aislamiento del paciente hasta 14 días después de la resolución del cuadro clínico. El algoritmo de confirmación considera el diagnóstico diferencial con otros patógenos respiratorios como influenza, parainfluenza, adenovirus, virus sincicial respiratorio, coronavirus del síndrome respiratorio de oriente medio (MERS-CoV), SARS-CoV y otros coronavirus. ${ }^{41}$

La situación actual de Covid-19 es de pronóstico reservado. El desconocimiento de la fuente de 


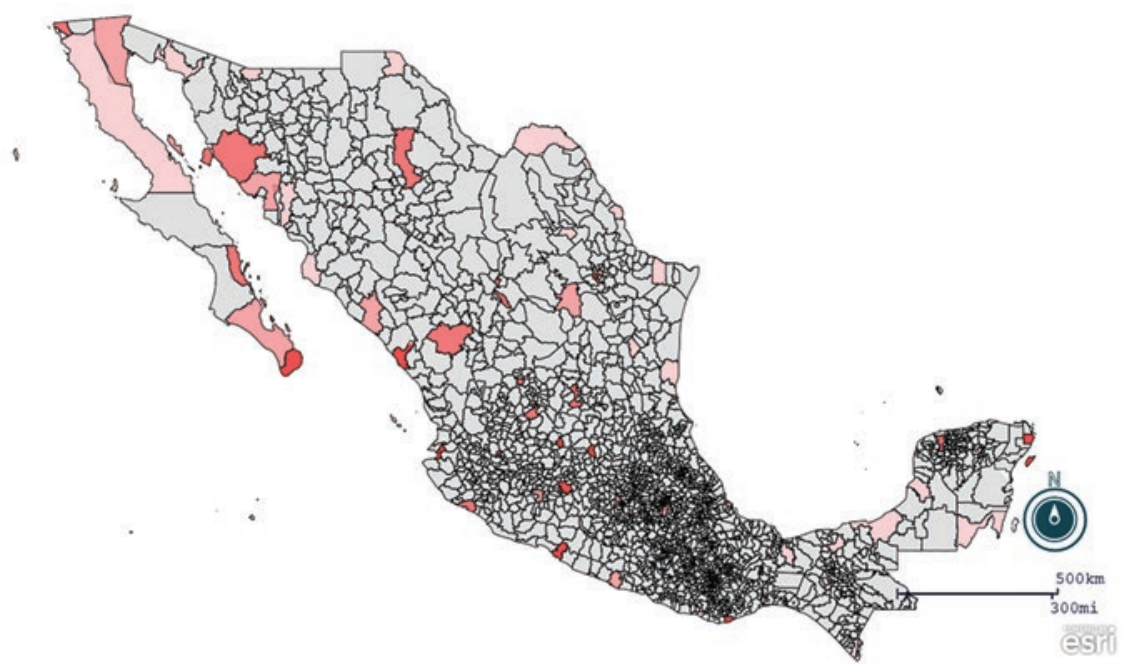

Figura 2. Coropleta que representa el número de visitantes de países con casos confirmados de Covid-19, por municipio, hasta noviembre de 2019. Datos de la Secretaría de Relaciones Exteriores.

dispersión, además de no tener tratamiento específico ni vacuna preventiva, nos coloca en un escenario desafiante en el que la colaboración, el intercambio de información y la transparencia entre los distintos sectores de la sociedad son valiosos medios de protección. ${ }^{42,43}$

Durante 2019, en México un total de 60 municipios (con 32851129 de habitantes) recibieron 15072653 visitantes por vía aérea de los países que hasta el momento han confirmado casos de Covid-19 (Figura 2). Lo anterior pone en perspectiva la conectividad de las distintas regiones del país, en su mayoría territorios densamente poblados. Estos destinos representarían las zonas de mayor riesgo para la introducción del patógeno.

\section{Consideraciones para los trabajadores de la salud}

Las epidemias anteriores han dejado lecciones útiles a la humanidad. En este momento del brote de Covid-19, la difusión efectiva de la información es clave para cortar las cadenas de transmisión. La OMS ha dedicado un sitio público a la difusión de cursos para el aprendizaje continuo que contiene módulos de actualización, tanto a nivel de respuesta en salud pública y clínica como de laboratorio. ${ }^{44-47}$

De forma coordinada, la academia e instituciones privadas han creado foros de discusión científica, plataformas de visualización y difusión de información del brote que permiten la trazabilidad de los eventos en tiempo real. Aunque no siempre coincidirán con las cifras oficiales, estas herramientas representan una modalidad para el estudio del brote que la salud pública no había utilizado hasta la fecha. ${ }^{14,46,48,49}$

\section{Conclusiones}

La estrecha dinámica que tenemos con otras especies ha provocado la emergencia de epidemias relacionadas con patógenos desconocidos o que pensábamos bajo control. En términos de salud global es difícil delimitar nacionalidades y territorios. El brote de Covid-19 dejará lecciones sobre el poder que la colaboración tiene ante una ESPII, independientemente de cuántos países alcance a afectar.

En México debemos aprender de las lecciones y estrategias de los países que han sido afectados. Todos los días se genera conocimiento nuevo que, si se utiliza adecuadamente, nos permitirá enfrentar la introducción de este y otros patógenos emergentes.

\section{Conflicto de intereses}

Ninguno.

\section{Financiamiento}

La presente investigación no ha recibido ninguna beca específica de agencias de los sectores público, comercial, y se llevó a cabo sin ánimo de lucro.

\section{Referencias}

1. Cui J, Li F, Shi ZL. Origin and evolution of pathogenic coronaviruses. Nat Rev Microbiol. 2019;17(3):181-92. DOI: 10.1038/s41579-018-0118-9

2. Lu R, Zhao X, Li J, Niu P, Yang B, Wu H, et al. Genomic characterisation and epidemiology of 2019 novel coronavirus: implications for virus origins and receptor binding. Lancet. 2020;6736(20):1-10. Disponible en: http:// www.ncbi.nlm.nih.gov/pubmed/32007145

3. Hoffmann M, Kleine-Weber H, Krüger N, Müller M. The novel coronavirus 2019 (2019-nCoV) uses the SARS-coronavirus receptor ACE2 and the 
cellular protease TMPRSS2 for entry into target cells. bioRxiv. 2020 Jan 31. DOI: 10.1101/2020.01.31.929042.

4. Wan Y, Shang J, Graham R, Baric RS, Li F. Receptor recognition by novel coronavirus from Wuhan: an analysis based on decade-long structural studies of SARS. J Virol. 2020 Jan 29. pii: JVI.00127-20. DOI: 10.1128/JVI.00127-20. [Epub ahead of print]. Disponible en: http://www. ncbi.nlm.nih.gov/pubmed/31996437

5. Ramaiah A, Arumugaswami V. Insights into cross-species evolution of novel human coronavirus 2019-nCoV and defining immune determinants for vaccine development. bioRxiv. 2020 Feb 4. DOI: 10.1101/2020.01.29.925867

6. Lu H, Stratton CW, Tang Y. Outbreak of pneumonia of unknown etiology in Wuhan China: the mystery and the miracle. J Med Virol. 2020;jmv.25678 Disponible en: https://onlinelibrary.wiley.com/doi/abs/10.1002/jmv.25678

7. World Health Organization. SARS (severe acute respiratory syndrome) [Consultado 2020 Feb 1]. Disponible en: https://www.who.int/ith/diseases/sars/en

8. Li Q, Guan X, Wu P, Wang X, Zhou L, Tong Y, et al. Early transmission dynamics in Wuhan, China, of novel coronavirus-infected pneumonia. N Engl J Med. 2020;1-9. Disponible en: http://www.ncbi.nlm.nih.gov/ pubmed/31995857

9. World Health Organization. WHO guidelines for the global surveillance of severe acute respiratory syndrome (SARS). Updated recommendations, October 2004. Disponible en https://www.who.int/csr/resources/ publications/WHO_CDS_CSR_ARO_2004_1/en

10. World Health Organization. Middle East respiratory syndrome case definition for reporting to WHO. WHO 2017 Jul 26. Disponible en: https:/ www.who.int/csr/disease/coronavirus_infections/case_definition/ en/\%0Ahttp://www.who.int/csr/disease/coronavirus_infections/mers-interim-case-definition.pdf?ua=1

11. World Health Organization. Novel coronavirus 2019. [Consultado 2020 Feb 1]. Disponible en: https://www.who.int/emergencies/diseases/ novel-coronavirus-2019

12. World Health Organization. About IHR. WHO; 2017. [Consultado 2019 Ago 20]. Disponible en: https://www.who.int/ihr/about/en

13. Corman VM, Landt O, Kaiser M, Molenkamp R, Meijer A, Chu DK, et al Detection of 2019 novel coronavirus (2019-nCoV) by real-time RT-PCR. Eurosurveillance. 2020;25(3):2000045. Disponible en: https://www.eurosurveillance.org/content/10.2807/1560-7917.ES.2020.25.3.2000045

14. Nextstrain [sitio web]. Real-time tracking of pathogen evolution. [Consultado 2020 Feb 8]. Disponible en: https://nextstrain.org

15. Cohen J. New coronavirus threat galvanizes scientists. Science. 2020;367(6477):492-493.

16. Du Toit A. Outbreak of a novel coronavirus. Nat Rev Microbiol. 2020;41579. Disponible en: http://dx.doi.org/10.1038/s41579-020-0332-0

17. Phan LT, Nguyen T V, Luong QC, Nguyen T V, Nguyen HT, Le HQ, et al. Importation and human-to-human transmission of a novel coronavirus in Vietnam. N Engl J Med. 2020 Jan 28. [Epub ahead of print]. DOI: 10.1056/NEJMc2001272.

18. World Health Organization. Novel coronavirus (2019-nCoV). Situation report 1. 21 January 2020. WHO; 2020. Disponible en: https://www.who. int/docs/default-source/coronaviruse/situation-reports/20200121-sitrep-12019-ncov.pdf?sfvrsn=20a99c10_4

19. Chinazzi M, Davis JT, Gioannini C, Pastore A, Rossi L, Xiong X, et al. Preliminary assessment of the international spreading risk associated with the 2019 novel coronavirus (2019-nCoV) outbreak in Wuhan city. Center for Inference \& Dynamics of Infectious Diseases [sitio web]; 2020. Disponible en: http://www.cidid.org/publications-1/2020/1/20/preliminary-assessment-of-the-international-spreading-risk-associated-with-the-2019-novel-coronavirus-2019-ncov-outbreak-in-wuhan-city

20. Bogoch II, Watts A, Thomas-Bachli A, Huber C, Kraemer MUG, Khan K. Potential for global spread of a novel coronavirus from China. J Trave Med. 2020 Jan 27;53(9):1689-99. DOI: 10.1093/jtm/taaa011/5716260

21. Isaac I. Bogoch, Alexander Watts, Andrea Thomas-Bachli, Carmen Huber, Moritz U.G. Kraemer KK. Pneumonia of Unknown Etiology in Wuhan, China: Potential for International Spread Via Commercial Air Travel. J Travel Med. 2020

22. Wu JT, Leung $K$, Leung GM. Nowcasting and forecasting the potential domestic and international spread of the 2019-nCoV outbreak originating in Wuhan, China: a modelling study. Lancet. 2020 Jan;6736(20). Disponible en: 10.1016/S0140-6736(20)30260-9

23. World Health Organization. Novel Coronavirus (2019-nCoV). Situation report-3. [Consultado 2020 Feb 8]. Disponible en: https://www.who.int/ docs/default-source/ coronaviruse/situation-reports/ 20200123-sitrep-32019-ncov, pdf?sfvrsn=d6d23643 8

24. Imai N, Dorigatti I, Cori A, Riley S, Ferguson NM. Estimating the potential total number of novel coronavirus cases in Wuhan City, China. Imp Coll London. 2020 Jan 17:1-4. Disponible en: https://www.imperial.ac.uk/ media/imperial-college/medicine/sph/ide/gida-fellowships/2019-nCoV -outbreak-report-17-01-2020.pdf

25. World Health Organization. Statement on the second meeting of the International Health Regulations (2005) Emergency Committee regarding the outbreak of novel coronavirus (2019-nCoV). [Consultado 2020 Feb 1] Disponible en: https://www.who.int/ news-room/detail/ 30-01-2020-state- ment-on-the-second-meeting-of-the-international-health-regulations-(2005)-emergency-committee-regarding-the-outbreak-of-novel-coronavirus -(2019-ncov)

26. World Health Organization. What are the International Health Regulations and Emergency Committees? [Consultado 2020 Jan 31]. Disponible en: https://www.who.int/news-room/q-a-detail/what-are-the-international -health-regulations-and-emergency-committees

27. Simón-Soria F. Public Health Emergencies of International Concern. An opportunity to improve global health security. Enferm Infecc Microbiol Clin. 2016;34(4):219-221.

28. World Health Organization. Surveillance case definitions for human infection with novel coronavirus (nCoV). WHO; 2020. Disponible en: https:// www.who.int/publications-detail/surveillance-case-definitions-for-human-infection-with-novel-coronavirus-(ncov)

29. World Health Organization. US\$675 million needed for new coronavirus preparedness and response global plan. [Consultado 2020 Feb 8]. Disponible en: https://www.who.int/ news-room/detail/05-02-2020-us-675-million-needed-for-new-coronavirus-preparedness-and-response-global-plan

30. World Health Organization. Novel coronavirus(2019-nCoV). Situation report-17. [Consultado 2020 Feb 9]. Disponible en: https://www.who.int/ docs/ default-source/coronaviruse/ situation-reports/20200206 -sitrep-17ncov.pdf? sfvrsn=17f0dca 4

31. World Health Organization. Novel coronavirus (2019-nCoV). Situation report 11. WHO Bull. 2020 Jan 31:1-7.

32. Strzelecki A. Infodemiological study using Google trends on coronavirus epidemic in Wuhan, China. 2020. arXiv. 2001:11021. Disponible en: http://arxiv.org/abs/2001.11021

33. World Health Organization. Novel coronavirus (2019-nCov). Situation report 20. WHO; 2020. p. 7 [Consultado 2020 Feb 9]. Disponible en: https://www.who.int/docs/default-source/coronaviruse/situation-reports/20200209-sitrep-20-ncov.pdf?sfvrsn=6f80d1b9_4

34. Tao Liu, Jianxiong Hu, Min Kang, Lifeng Lin, Haojie Zhong, Jianpeng Xiao GH. Transmission dynamics of 2019 novel coronavirus (2019-nCoV). bioRxiv. 2020;21(1):1-9. DOI: https://doi.org/10.1101/2020. 01.25.919787

35. Read JM, Bridgen JR, Cummings DA, Ho A, Jewell CP. Novel coronavirus 2019-nCoV: early estimation of epidemiological parameters and epidemic predictions. medRxiv. 2020 Dec 28. DOI: 10.1101/ 2020.01.23.20018549

36. Worldometer [sito web]. Wuhan Coronavirus outbreak. [Consultado 2020 Feb 9]. Disponible en: https://www.worldometers.info/coronavirus

37. Huang C, Wang Y, Li X, Ren L, Zhao J, Hu Y, et al. Clinical features of patients infected with 2019 novel coronavirus in Wuhan, China. Lancet. 2020;6736(20):1-10. Disponible en: https://doi.org/10.1016/S01406736(20)30183-5

38. Secretaría de Salud. Nuevo coronavirus 2019 nCoV-Comunicado técnico diario. [Consultado 2020 Feb 1]. Disponible en: https://www.gob.mx/ salud/documentos/nuevo-coronavirus-2019-ncov-comunicado-tecnico-diario

39. Secretaría de Salud. Lineamiento estandarizado para la vigilancia epidemiológica y por laboratorio de enfermedad por 2019-nCov. Disponible en: http://cvoed.imss.gob.mx/ secretaria-de-salud-lineamiento-estandarizado-para-la-vigilancia-epidemiologica-y-por-laboratorio-de-enfermedad-por-201-ncov/\# iLightbox[gallery2287]/0

40. Dirección General de Epidemiológica S de S. Lineamiento estandarizado para la vigilancia epidemiología y por laboratorio de enfermedad por 2019nCov_7_2_2020. 2020.

41. Lu C, Liu X, Jia Z. 2019-nCoV transmission through the ocular surface must not be ignored. Lancet. 2020 Feb 6. DOI: 10.1016/S0140-6736(20)30313-5

42. Secretaría de Salud. Lineamientos para la vigilancia por laboratorio del denque y otras arbovirosis. México: SSa; 2017. p. 12-95.

43. World Health Organization. National capacities review tool for a novel coronavirus (nCoV). WHO; 2020. Disponible en: https://www.who.int/ publications-detail/national-capacities-review-tool-for-a-novel-coronavirus-(ncov)

44. World Health Organization. Risk communication and community engagement (RCCE) readiness and response to the 2019 novel. WHO; 2020 Jan 26. Disponible en: https://www.who.int/publications-detail/ risk-communication-and-community-engagement-readiness-and-initialresponse-for-novel-coronaviruses-(-ncov)

45. OpenWHO [sitio web]. Trainings for current outbreaks. Covel coronavirus (2019-nCoV). [Consultado 2020 Feb 9]. Disponible en: https://openwho.org

46. World Health Organization. Critical care severe acute respiratory infection training. [Consultado 2020 Feb 9]. Disponible en: https://openwho. org/courses/ severe-acute-respiratory-infection

47. World Health Organization. Technical guidance novel coronavirus 2019. [Consultado 2020 Feb 9]. Disponible en: https://www.who.int/emergencies/ diseases/novel-coronavirus-2019/ technical-guidance

48. Rambaut A. Phylogenetic analysis of $23 \mathrm{nCoV}-2019$ genomes. Virological [Internet]. 2020 Ene 23. Disponible en: http://virological.org/t/phylogenetic-analysis-of-23-ncov-2019-genomes-2020-01-23/335

49. Engineering JHWS of. Coronavirus 2019-nCoV [Internet]. 2020 [Consultado 2020 Feb 1]. Disponible en: https://gisanddata.maps.arcgis.com/ apps/opsdashboard/index.html\#/bda7594740fd40299423467b48e9ecf6 